OPEN ACCESS

Edited by: Mats Bemark,

University of Gothenburg, Sweden

Reviewed by:

Anja Joachim,

Veterinärmedizinische Universität,

Austria

Virginia Marugan-Hernandez,

Royal Veterinary College, UK

*Correspondence:

Xun Suo

suoxun@cau.edu.cn

tThese authors have contributed equally to this work.

Specialty section:

This article was submitted to

Mucosal Immunity,

a section of the journal

Frontiers in Immunology

Received: 15 October 2016 Accepted: 03 January 2017

Published: 23 January 2017

Citation:

Tao G, Shi T, Tang $X$, Duszynski DW,

Wang Y, Li C, Suo J, Tian X, Liu X and Suo $X$ (2017) Transgenic Eimeria magna Pérard, 1925 Displays Similar

Parasitological Properties to the

Wild-type Strain and Induces an Exogenous Protein-Specific Immune

Response in Rabbits (Oryctolagus cuniculus L.).

Front. Immunol. 8:2.

doi: 10.3389/fimmu.2017.00002

\section{Transgenic Eimeria magna Pérard, 1925 Displays Similar Parasitological Properties to the Wild-type Strain and Induces an Exogenous Protein- Specific Immune Response in Rabbits (Oryctolagus cuniculus L.)}

\author{
Geru Tao ${ }^{1,2+}$, Tuanyuan Shi ${ }^{3 \dagger}$, Xinming Tang ${ }^{1,2}$, Donald W. Duszynski ${ }^{4}$, Yunzhou Wang ${ }^{1,2}$, \\ Chao Li $^{1,2}$, Jingxia Suo ${ }^{1,2}$, Xiuling Tian ${ }^{1,2}$, Xianyong Liu ${ }^{1,2}$ and Xun Suo ${ }^{1,2 *}$

\begin{abstract}
${ }^{1}$ State Key Laboratory of Agrobiotechnology, China Agricultural University, Beijing, China, ${ }^{2}$ National Animal Protozoa Laboratory \& College of Veterinary Medicine, China Agricultural University, Beijing, China, ${ }^{3}$ Department of Animal Parasitology, Institute of Animal Husbandry and Veterinary Medicine, Zhejiang Academy of Agricultural Science,
\end{abstract} \\ Hangzhou, China, ${ }^{4}$ Department of Biology, University of New Mexico, Albuquerque, NM, USA
}

Rabbit coccidiosis causes great economic losses to world rabbitries. Little work has been done considering genetic manipulation on the etiological agents, rabbit Eimeria spp. In this study, we constructed a transgenic line of Eimeria magna (EmagER) expressing enhanced yellow fluorescent protein (EYFP) and red fluorescent protein (RFP) using regulatory sequences of Eimeria tenella and Toxoplasma gondii. We observed the life cycle of EmagER and confirmed that the transgenic parasites express exogenous proteins targeted to different cellular compartments throughout the entire life cycle. EYFP was expressed mainly in the nucleus and RFP both in the nucleus and cytoplasm. Then, coccidia-free, laboratory-reared 40-day-old rabbits were primarily infected with either EmagER or wild-type strain oocysts and challenged with the wild-type strain. EmagER showed similar reproductivity and immunogenicity to the wild-type strain. Finally, we examined the foreign protein-specific immune response elicited by EmagER. Rabbits were immunized with either transgenic or wild-type oocysts. Immune response against parasite-soluble antigen, EYFP and RFP in spleen, and mesenteric lymph nodes were detected by quantitative real-time PCR. The relative expression level of IFN- $\gamma, \mathrm{IL}-2$, and TNF- $\alpha$ were higher in EmagER-immunized rabbits than wild-type parasites-immunized rabbits after stimulation with EYFP and RFP. Our study confirmed that a specific immune response was induced by the exogenous protein expressed by EmagER and favored future studies on application of transgenic rabbit coccidia as recombinant vaccine vectors.

Keywords: rabbit coccidia, transgenic coccidia, Eimeria magna, life cycle, immunity 


\section{INTRODUCTION}

Eimeria spp. are obligate intracellular parasites that infect epithelial cells of a particularly wide range of vertebrate species (1). Severe infections with Eimeria spp. can result in a serious disease, coccidiosis, which causes huge economic losses in two primary food animal industries, namely poultry and rabbitry (2-4).

Genetic manipulation of chicken Eimeria spp. has been an active and ongoing area of research for almost two decades (5) because it can be a strong tool toward understanding gene function and intimate interaction between these parasites and their hosts. To date, several transgenic chicken Eimeria lines have been developed. Besides Eimeria tenella (6), successful stable transfection of Eimeria mitis also was developed. Recently, Qin et al. (7) demonstrated that enhanced yellow fluorescent protein (EYFP) expressed by transgenic E. mitis contributed to a more detailed observation of this parasite's endogenous stages. Not only for exploration on parasites' biological characteristics, transgenic Eimeria parasites have great potential to be utilized as live recombinant vaccine vectors. Previous studies demonstrated that the exogenous protein expressed by transgenic parasites stimulate specific immune responses in the host. One study (8) showed that immunization with a transgenic E. mitis line expressing chicken IL-2 stimulated an enhanced cellular immune response against it. In addition, a transgenic line of E. tenella expressing Toxoplasma gondii SAG1 protein (TgSAG1) elicits protective immunity against T. gondii in chicken and mice (9). All these reports reinforced the broad prospect of transgenesis of Eimeria spp.

Similar work with rabbit Eimeria spp., however, lags far behind what we know about chicken coccidia. Eimeria magna is a common species of rabbit coccidia often found in great numbers on rabbit farms (11). It locates in the small intestine and produces a moderate pathogenicity and immunogenic response in rabbits $(12,13)$. In this study, we constructed a transgenic line of E. magna (EmagER) expressing double reporter genes, EYFP (7) and red fluorescent protein (RFP). Further investigations of its life cycle, reproductivity, and immunogenicity were conducted to provide more information on transgenesis in rabbit coccidia and evaluate the potential capacity as vaccine vector.

\section{MATERIALS AND METHODS}

\section{Ethics Statement}

All experimental procedures were approved by the China Agricultural University Animal Ethics Committee (certified by Beijing Laboratory Animal employee, ID: 1114120800096), and due attention was paid to the welfare of the animals.

The rabbits were reared under stress-free environment, eliminating strong light and noise, with one rabbit per cage. Physical condition was monitored every day during all experimental procedures. Euthanasia was performed with an intracardiac pentobarbital overdose in accordance with the experiment design (14).

\section{Plasmid, Parasites, and Animals}

Plasmid used in the transfection, $p$ HDEp2aRA, is a single expression cassette where DHFR-Ts $2 \mathrm{~m} 3 \mathrm{~m}$, a pyrimethamine resistance gene from T. gondii, EYFP, and RFP were inserted between histone 4 promoter with its nuclear localization signal (90 bp) (15) and 3' untranslated region of actin of E. tenella. A porcine teschovirus-1 $2 \mathrm{~A}$ peptide (P2A) (66 bp), which was shown to be able to cleave two contiguous proteins $(10,16)$, was added between EYFP and RFP. Signal sequence of dense granule 8 (gran8) from T. gondii (84 bp) (17) was added to regulate secretion of RFP, which was followed by a His-tag (Figure 1A).

The wild-type E. magna (EmagWT) was originally isolated from Hebei province, China. Newly collected parasites were purified by flotation with saturated salt water followed by washing with sterilized water and incubation with 5\% sodium hypochlorite to eliminate most of the bacterial contamination. Purified parasites, both wild-type and transgenic strain, were propagated in coccidia-free rabbits and maintained in $2.5 \% \mathrm{w} / \mathrm{v}$ potassium dichromate at $4^{\circ} \mathrm{C}$ according to a standard protocol (18). For propagation of the parasites, 200 sporulated oocysts per rabbit were orally inoculated. Oocysts were collected from feces of 7-10 days post inoculation (dpi). Moreover, fluorescent sporulated oocysts of the transgenic strain screened by the fluorescent-activated cell sorting (FACS) were used in propagation or other assays.

All animals used in this study were obtained from Xinglong Laboratory Animal Breeding Center. To avoid coccidial contamination, 3-week-old New Zealand white rabbits were weaned and reared by bottle-feeding of powdered milk under coccidia-free condition away from their mothers till 4 weeks old. Also, 35- to 40-day-old rabbits for parasite propagation and other assays were kept in isolators and fed with coccidia-free pellet feed and water ad libitum.

\section{Transfection of E. Magna, Selection, and Propagation of EmagER}

Transfection of E. magna sporozoites was conducted according to an established protocol (6). Briefly, $2 \times 10^{7}$ purified sporozoites were electroporated using a nucleofector (Program U-033, AMAXA, Switzerland) by a restriction enzyme-mediated method in a system containing $100 \mu \mathrm{l}$ cytomix buffer, $15 \mu \mathrm{g}$ linearized DNA, and 250 UI SnaBI restriction enzyme (15). Sporozoites were resuspended in DMEM and inoculated to MDBK culture for in vitro transient transfection or injected into duodenum of a 4-week-old rabbit in a laparotomy for in vivo stable transfection. In stable transfection, oocysts from feces of 7-10 dpi were collected as described above. Screening of the transgenic oocysts was conducted by FACS (MoFlo Cell Sorter, Dako-Cytomation, Fort Collins, CO, USA) and addition of $150 \mathrm{mg} / \mathrm{kg}$ pyrimethamine in the rabbit pellet as a drug selection was performed.

\section{Genomic and Expression Analysis of Exogenous Genes of EmagER}

Integration site and expression of the exogenous DNA were investigated by genome walking analysis and Western blot. First, the flanking sequences of the $5^{\prime}$ integration site were identified using a genome walking kit (Takara, China). EmagER genomic DNA was obtained by phenol/chloroform extraction from sporozoites. Specific primers were obtained according to E. tenella His4 promoter sequence as previously described (7). PCR products of 


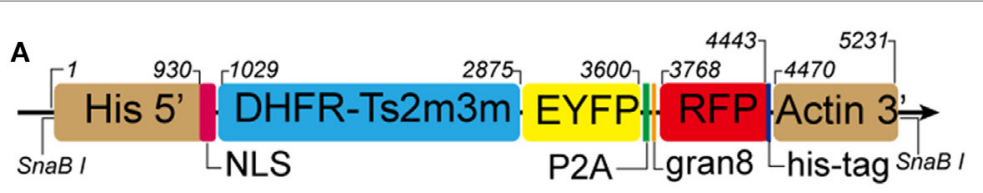

B

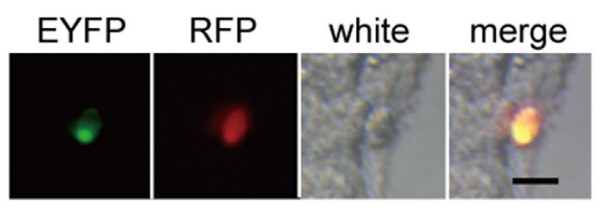

C

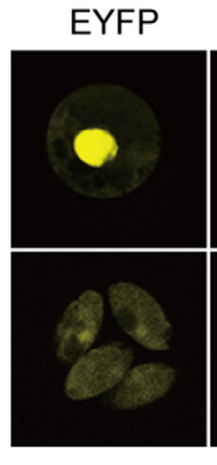

RFP
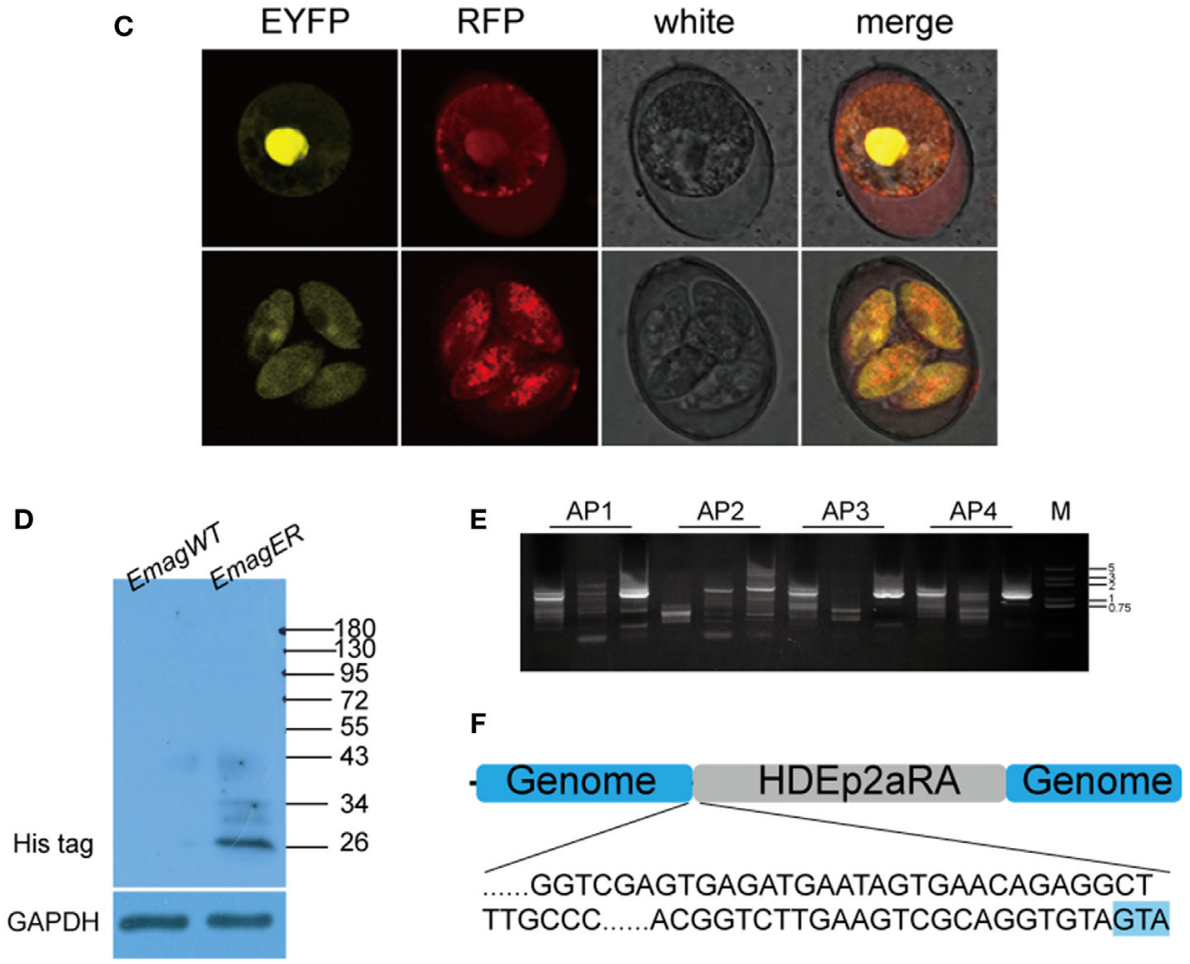

FIGURE 1 | Construction and identification of transgenic Eimeria magna-expressing enhanced yellow fluorescent protein (EYFP) and red fluorescent protein (RFP) (EmagER). (A) Schematic diagram of transfected linearized plasmid HDEp2aRA. DHFR-Ts2m3m fused with EYFP and RFP were inserted between promoter of histone 4-NLS and 3' UTR of actin from Eimeria tenella. NLS, nuclear localization signal of histone 4; P2A, porcine teschovirus-1 2A peptide; gran8, signal sequence of Toxoplasma gondii dense granule 8. (B) Transient transfection of $E$. magna sporozoites in MDBK culture. Freshly purified sporozoites were electroporated with $15 \mu \mathrm{g}$ pHDEp2aRA and inoculated into MDBK cell culture. Fluorescent sporozoites were observed $20 \mathrm{~h}$ post inoculation. Bar $=10 \mu \mathrm{m}$. (C) Unsporulated and sporulated oocysts of EmagER. EYFP was mainly located in the nucleus and RFP in the nucleus and the cytoplasm. (D) Western blot identifying P2A function in EmagER. Soluble abstract of EmagWT and EmagER were subjected to SDS-PAGE and Western blot using mAb against His-tag. A polyclonal antibody against GAPDH was included as a loading control. (E,F) Genome walking analysis identified the $5^{\prime}$ insertion site of the transfected plasmid. GTA in blue shading indicated cleaved linearization site (SnaBl).

the last round were recovered and cloned into pEASY-T1-simple vector (TransGen Biotech), sequenced, and the results were analyzed by DNAStar 7.0 software. Twelve insertion site sequencing were conducted. Second, we applied Western blot to identify the "self-cleaving" effectiveness of P2A in transgenic parasites. Whole parasite soluble extracts were prepared from sporozoites as previously described (19). The lysis was centrifuged at 10,000 rpm for $10 \mathrm{~min}$, and the supernatant was then subjected to SDS-PAGE and Western blot. Monoclonal antibody against His-tag (ABclonal) was applied. E. tenella GAPDH polyclonal antibody obtained and preserved in our lab was included as a loading control to ensure similar amounts of parasites' soluble antigen were applied.

\section{Observation on Whole Life Cycle of EmagER}

Seven 4-week-old rabbits were orally inoculated with sporulated oocysts of EmagER from the fifth passage and euthanized at 24, $48,72,84,120,144$, and $152 \mathrm{~h}$ post inoculation (hpi) $(n=1$ animal per time point) as described before (13). Jejunum and ileum were washed with cold HBSS, and smears were made by 
scraping the mucosa of the intestine. Fresh smears and sporulation process of newly collected oocysts were visualized under a confocal laser scanning microscopy (SP5, Leica, Germany) for observation of different developmental stages of fluorescent parasites.

\section{Reproductivity and Immunogenicity of EmagER}

To explore the biological features of the transgenic strain, reproductivity and immunogenicity compared with the parental strain (EmagWT) were investigated. Sixteen 40-day-old rabbits housed one per cage were randomly distributed into four groups with four rabbits per group. Two groups of rabbits were orally inoculated either with 200 oocysts of EmagWT or EmagER. The third group was not immunized and applied as an unimmunized challenged control. The fourth group was applied as an unimmunized and unchallenged control (UUC). Daily oocyst outputs during patent period (6.5-12 dpi) of each infected rabbit were counted according to a standard McMaster technique (18). Also, $14 \mathrm{dpi}$, a challenge with $1 \times 10^{4}$ sporulated oocysts of EmagWT was performed to each rabbit except for the ones in the UUC group. Oocyst outputs of all challenged rabbits were counted. Clinical signs of experimental animals were monitored every day, and body weight was measured twice a week during the whole experimental process.

\section{Immunization and Real-time PCR}

To investigate the exogenous protein-specific immunity stimulated by the transgenic parasites, fifteen 35-day-old coccidia-free rabbits were randomly distributed into three groups. Two groups of rabbits were inoculated with 200 oocysts of either EmagWT or EmagER. The last group of rabbits was inoculated with $200 \mu \mathrm{l}$ PBS as control. Fourteen days later, all rabbits were euthanized. Splenocytes and mesenteric lymph nodes (MLN) lymphocytes were isolated, and single cell suspensions were prepared. Lymphocytes were adjusted to $5 \times 10^{6} / \mathrm{ml}$ and grown in RPMI1640 culture medium supplemented with fetal bovine serum $[10 \%(\mathrm{v} / \mathrm{v})]$, penicillin $(200 \mathrm{U} / \mathrm{ml})$-streptomycin (streptomycin) at $37^{\circ} \mathrm{C}$ in an atmosphere of $5 \% \mathrm{CO}_{2}$. EmagWT-soluble antigen (EmagSA) $(20 \mu \mathrm{g} / \mathrm{ml})$, recombinant EYFP (rEYFP) $(20 \mu \mathrm{g} / \mathrm{ml})$, and recombinant RFP (rRFP) $(20 \mu \mathrm{g} / \mathrm{ml})$ were added separately as in vitro stimulus. A cell stimulation cocktail containing PMA $(40.5 \mu \mathrm{M})$ and ionomycin $(670 \mu \mathrm{M})$ (eBioscience) was used as a positive stimulus. Lymphocytes were harvested $12 \mathrm{~h}$ after stimulation, washed twice with sterilized PBS, and total RNA was extracted using TRIzol reagent (Invitrogen, Shanghai). Total cDNA was synthesized using an EasyScript First-Strand cDNA Synthesis SuperMix (Transgen, China). The primer pairs used for analysis of four specific genes (Table 1) were designed through NCBI Primer Designing Tool (http://www.ncbi.nlm.nih.gov/ tools/primer-blast/). Quantitative real-time PCR was performed using a SYBR ${ }^{\circledR}$ Premix Ex Taq ${ }^{\mathrm{TM}}$ (Takara) on 7500 Real-Time PCR System (Applied Biosystems) with a program of $50^{\circ} \mathrm{C}$ for $2 \mathrm{~min}, 95^{\circ} \mathrm{C}$ for $10 \mathrm{~min}, 40$ cycles of $95^{\circ} \mathrm{C}$ for $15 \mathrm{~s}$, and $60^{\circ} \mathrm{C}$ for 1 min. Relative gene expression was calculated by the $2^{-\Delta \Delta C q}$ (Livak) method (20).

\section{Statistical Analysis}

Statistical analysis was performed by one-way ANOVA using the SPSS 19.0 software. Data was presented as mean \pm SEM. Difference between groups were considered statistically significant when $p$ values were less than 0.05 .

\section{RESULTS}

\section{Construction and Identification of a Transgenic Line of E. Magna Expressing EYFP and RFP (EmagER)}

For in vitro transfection, fluorescent sporozoites in MDBK cell culture were observed $20 \mathrm{~h}$ after inoculation (Figure 1B). As the foreign genes were initiated by His4-NLS, EYFP was mainly expressed in the nuclei of the sporozoites. Meanwhile, RFP was found both in the nuclei and the cytoplasm indicating that the transfected plasmid can be employed by E. magna. After several trials on in vivo transfection, we obtained a transgenic population at a transfection efficiency of $0.06 \%$. The fluorescent rate increased to $40 \%$ after five cycles of FACS and propagation in coccidia-free rabbits. Unfortunately, we did not obtain higher fluorescent rate in subsequent passages.

All transgenic oocysts express EYFP in the nuclei and RFP both in the nuclei and cytoplasm (Figure 1C). To further determine the function of P2A in EmagER, Western blot using an mAb against His-tag, which was ligated to RFP in the transfection plasmid, was performed. A $26 \mathrm{kDa}$ (RFP-6 $\times$ His) instead of a $128 \mathrm{kDa}$ (DHFREYFP-RFP- $6 \times$ His) band was detected demonstrating that P2A was efficient as self-cleaving peptide in E. magna (Figure 1D). Genome walking analysis of the oocysts from fifth passages were conducted (Figure 1E), and the $5^{\prime}$ integration site was determined (Figure 1F). Collectively, we obtained a transgenic E. magna strain expressing EYFP and RFP.

\section{EmagER Expresses Exogenous Proteins Targeted to Different Cellular Compartment throughout the Entire Life Cycle}

By taking advantages of the fluorescent proteins expressed by the transgenic parasites, the morphological features of transgenic parasites during all developmental stages were observed (Figure 2). Invading sporozoites as well as trophozoites ongoing nucleus division were found at $24 \mathrm{hpi}$ (Figures 2A,B). Mature meronts of each generation were found at 48, 66, 102, and $120 \mathrm{hpi}$, respectively (Figures $2 \mathbf{C}-\mathbf{M}$ ). Since EYFP was mainly expressed in the nuclei, both multinucleate and uninucleate merozoites were vividly distinguishable. Mature microgametocytes containing thousands of microgametes (Figure 2N) and macrogametocytes whose EYFP was not only located within the large nucleus but also Golgi adjunct (21) (Figure 20) were discovered during 144-152 hpi. During the whole endogenous stages, the transgenic parasites express EYFP in the nucleus and RFP in the nucleus and cytoplasm.

In addition, sporogony of the transgenic parasites was also observed (Figure 3). Oocysts of the transgenic strain, freshly 
TABLE 1 | Primer pairs for quantitative real-time PCR.

\begin{tabular}{|c|c|c|c|}
\hline Target gene & Forward and reverse primer sequences $5^{\prime}-3^{\prime}$ & Target size (bp) & NCBI accession \\
\hline $\mathrm{IFN}-\gamma$ & $\begin{array}{l}\text { F: GCTCTGCCTCATCTTGGGTT } \\
\text { R: GGTCCACCATTTGCCACATC }\end{array}$ & 117 & NM_001081991.1 \\
\hline IL-2 & $\begin{array}{l}\text { F: GCCCAAGAAGGTCACAGAATTG } \\
\text { R: TGCTGATTGATTCTCTGGTATTTCC }\end{array}$ & 128 & NM_001163180 \\
\hline TNF- $\alpha$ & $\begin{array}{l}\text { F: AGCCCACGTAGTAGCAAACC } \\
\text { R: TGAGTGAGGAGCACGTAGGA }\end{array}$ & 192 & NM_001082263.1 \\
\hline
\end{tabular}

Primer pairs were designed within the coding sequences (CDS) of rabbit genes available at NCBI. All primers were designed using Primer-BLAST and made to span exon junctions when possible.

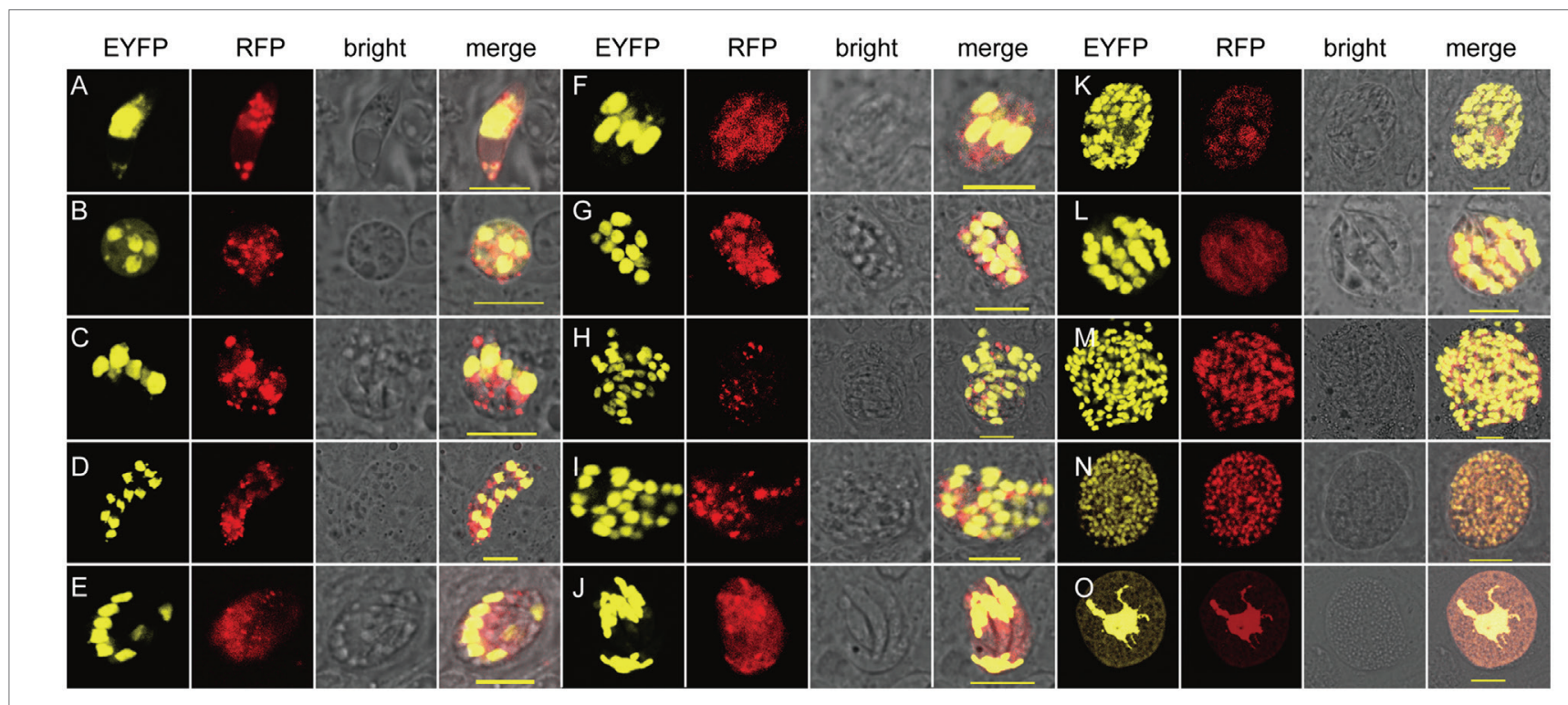

FIGURE 2 | Exogenous proteins were expressed and targeted to different cellular compartments in endogenous developmental stages of EmagER. (A) A sporozoite at $24 \mathrm{hpi}$ (B) a trophozoite at 24 hpi; (C-M) first to fourth generations of meronts of both type A (multinucleate) and type B (uninucleate) meronts at 48-120 hpi; (N) a microgametocyte at $152 \mathrm{hpi}$; and (O) a macrogametocyte at $144 \mathrm{hpi}$; bar $=20 \mu \mathrm{m}$.

collected from fecal samples and purified by floatation with saturated salt water were applied (Figure 3A). The first nuclear division proceeded after contraction or shrinkage of the cytoplasmic mass (Figures 3B,C). Completion of the second nuclear division and cytokinesis was evidenced by four sporoblasts symmetrically projected from the central cytoplasmic mass (Figure 3D) and soon developed into four separated spheres and an oocystic residua (Figure 3E). During all three nuclear division and two cytokinesis, EYFP was located in the nucleus and RFP mainly in the cytoplasm (Figure 3). Observation of all the developmental stages of the transgenic parasites demonstrated that the exogenous proteins were expressed and targeted to different cellular compartments.

\section{Reproductivity and Immunogenicity of EmagER}

To investigate the biological feature of EmagER, reproductivity and immunogenicity were evaluated. First, daily oocyst outputs of rabbits infected with either EmagWT (200 oocysts) or EmagER
(200 oocysts) were measured 6-12 dpi. Oocysts of both strains were first shed on $6.5 \mathrm{dpi}$, increased sharply, and reached the peak on 8 dpi (Figure 4A). EmagER presented slightly reduced reproductivity (mean \pm SEM, $\left.9.464 \times 10^{7} \pm 1.275 \times 10^{7}\right)$ compared with EmagWT $\left(\right.$ mean \pm SEM, $\left.1.057 \times 10^{8} \pm 2.244 \times 10^{7}\right)$. And no significant difference was found (one-way ANOVA, LSD). Next, a challenge of $1 \times 10^{4}$ oocysts of EmagWT was performed to all rabbits $14 \mathrm{dpi}$ in order to evaluate immunogenicity of the transgenic parasites. Robust immunity was evidenced, and no significant difference of body weight gain and oocyst reduction were observed compared with EmagWT (Figures 4B,C). Collectively, EmagER was of equal reproductivity and similar immunogenicity as EmagWT.

\section{Exogenous Proteins Expressed by EmagER Stimulated Local Immune Response}

The Th1 cytokine profile is a well-known indicator of cellular immunity elicited by intracellular pathogens including Eimeria 


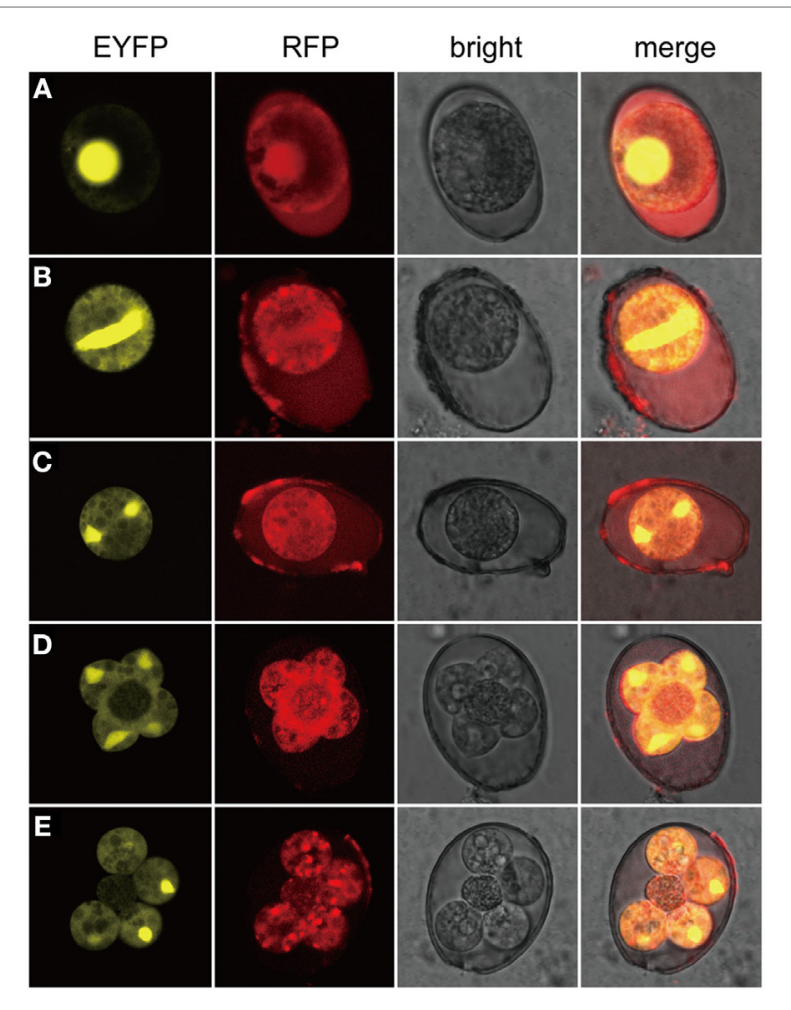

FIGURE 3 | Expression of exogenous proteins of EmagER during sporogony. Freshly collected oocysts of EmagER were incubated in $2.5 \%$ potassium dichromate at $27^{\circ} \mathrm{C}$ and observed at different time intervals under a confocal microscope (Leica, SP5, Germany). (A) A freshly collected unsporulated oocyst; (B) an oocyst during the process of spindle stage; (C) first nuclear division finished; (D) four separated sporoblasts projected from the central cytoplasmic mass during the second nuclear division; and (E) formation of four spheres of sporocysts and the oocystic residua.

spp. (22). To determine whether the exogenous proteins expressed by the transgenic parasites can stimulate specific immunity, we investigated the mRNA transcriptional level of Th1 cytokines of lymphocytes of immunized rabbits after an in vitro stimulation. Splenocytes and lymphocytes of MLN of experimental rabbits were isolated, stimulated, and mRNA transcriptional level of IFN- $\gamma$, IL- 2 , and TNF- $\alpha$ was analyzed by real-time PCR (primers were shown in Table 1). We found that the transcriptional level of IFN- $\gamma$, IL-2, and TNF- $\alpha$ of the splenocytes was relatively low, and there was no significant difference among immunized or non-immunized rabbits after in vitro stimulation (Figure 5). In MLN lymphocytes, on the other hand, EmagSA stimulation caused upregulated Th1 cytokine transcription of EmagWT- and EmagER-immunized rabbits. Furthermore, relative expression level of IFN- $\gamma$, IL-2, and TNF- $\alpha$ after stimulation with rEYFP and rRFP was higher in EmagER-immunized rabbits than that of other groups (Figure 5). This result indicated that EmagER stimulated exogenous protein-specific local immune response in rabbit.

\section{DISCUSSION}

The transgenesis of parasites in the phylum Apicomplexa has made great progress in recent years. In chickens, at least two
(E. mitis, E. tenella) of the seven most important Eimeria spp. have been transfected $(6,7)$. Much more detailed work in this area has been done with T. gondii (23), Plasmodium spp. (24), and Cryptosporidium spp. (25). In rabbits, however, the only work done on transgenesis was developed by Shi et al. (26) in a strain of Eimeria intestinalis. Here we report, for the first time, the construction of a transgenic line of E. magna, from the domestic rabbit, which expresses the dual reporter genes, EYFP and RFP. More importantly, the exogenous proteins expressed by this transgenic parasite stimulated a specific local immune response in our rabbits. This discovery in rabbits offers the prospect that transgenic rabbit coccidia might be candidates to transport other proteins as recombinant biological vaccines.

Here, we used the regulatory sequences of E. tenella, which was shown to be reliable in a previous study on transfection of $E$. intestinalis (26) in a plasmid generated by Tang et al. (10). This reliability might result from the functional conservation of $E$. tenella histone 4 and actin genes among these Eimeria species. Additionally, Tang et al. (10) provided an in-depth study on E. tenella demonstrating the "self-cleavage" peptide $2 \mathrm{~A}$ from porcine teschovirus-1. We also found that it was efficient in our transgenic E. magna. The regulator sequences and the "selfcleavage" peptide are simple, and efficient molecular tools may promote additional studies that will be useful in developing transgenic models with Eimeria species in any species.

As noted earlier, successful transfection studies have been accomplished with two species of chicken coccidia. Liu et al. (15) achieved $0.2 \%$ transfection rate with E. tenella. Later, Qin et al. (7) showed a similar rate $(0.19 \%)$ for E. mitis. The first study on transfection with rabbit coccidia by Shi et al. (26) involved E. intestinalis, but they succeed only $0.01 \%$ in their first passage. This lower transfection rate might be due to a higher susceptibility of mammalian sporozoites to electroporation than are those of bird coccidia for some, as yet, unknown reasons. In our study, the transfection rate in our first passage of E. magna was similar $(0.06 \%)$. Unfortunately, this is where the similarity ends between these two rabbit Eimeria spp. Shi et al. (26) demonstrated that the transgenic parasites grew up to $80 \%$ in the third passage by selection of FACS and propagation, whereas we obtained only $40 \%$ fluorescent parasites even with a system of DHFR-Ts $2 \mathrm{~m} 3 \mathrm{~m}$ after five passages. As the plasmid was randomly integrated into the parasite's genome, the site of integration may affect the transcription rate of the gene of interest (27). Unfortunately, we cannot confirm this due to the lack of an annotated reference genomic library.

In spite of what some might perceive as relatively low transfection success, our constitutive expression of fluorescent proteins presented major advantages that allowed us to identify developmental stages of whole life cycle of E. magna. Similar findings also were stated in research on transgenic E. mitis (7) in chickens and E. intestinalis (26) in rabbits. The major advantage of our procedures, however, is that we transfected dual reporter genes that targeted different cellular compartments. That is, EYFP is expressed mainly in the nuclei of all developmental stages of E. magna and the RFP targeted to the cytoplasm. Subcellular localization of RFP in EmagER was different from a previous 

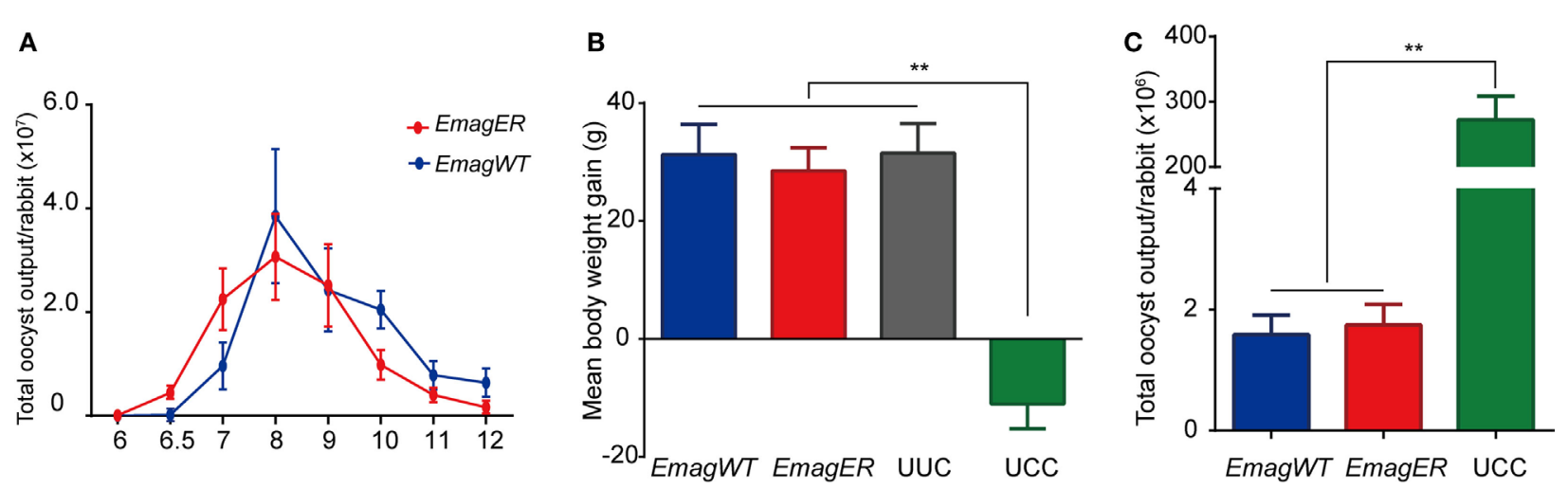

FIGURE 4 | Reproductivity and immunogenicity of EmagER. Three groups of rabbits were inoculated with either 200 oocysts of Eimeria magna wild-type (EmagWT), transgenic E. magna-expressing enhanced yellow fluorescent protein and red fluorescent protein (EmagER), or $200 \mu \mathrm{l}$ PBS, respectively. (A) Daily oocyst output of individual rabbit during patent period were measured using a McMaster counting chamber. Fourteen days later, a challenge of $1 \times 10^{4}$ oocysts of EmagWT was performed to all rabbits. Body weight gain (B) and total oocyst output (C) were measured in 14 days post challenge. UUC, unimmunized and unchallenged control; UCC, unimmunized and challenged control. All data were presented as mean \pm SEM values. Statistical analysis was performed by one-way ANOVA, LSD, ${ }^{\star} p<0.05$, and ${ }^{* *} p<0.01$.

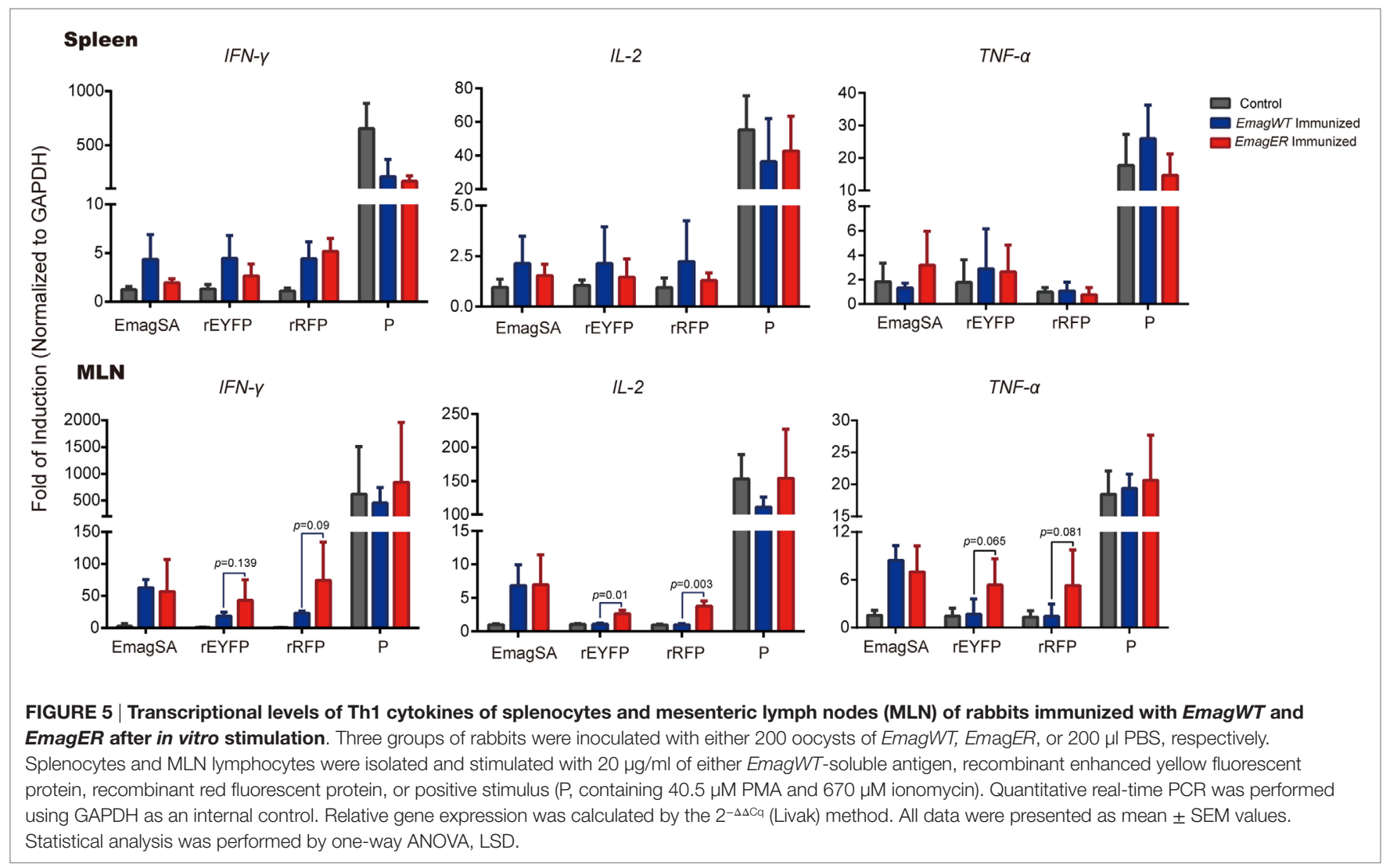

study on E. tenella where RFP was also regulated by gran8 yet secreted into the parasitophorous vacuole (17). Nevertheless, in the future, we could engineer other proteins, using the same vector, to deliver new antigens into the organelles of the various endogenous stages of rabbit coccidia, and perhaps other coccidia of domesticated mammals.
We did not observe significant systemic immune response as measured by cultured splenocytes, probably due to the low sensitivity of the test. However, a significant local immunity in the MLN was detected. Previous work in our laboratory (8) on transgenic chicken Eimeria demonstrated that enhanced cellular immunity was detected in PBMC of chickens immunized 
with transgenic parasites expressing chicken IL-2. However, the systemic immune response, at least in our rabbits, was low after primary infection of EmagWT or EmagER, consistent with a previous study on the dynamics of T-lymphocytes in rabbits immunized with $E$. intestinalis (28). In fact, enhanced transcriptional levels of Th1 cytokines in MLN, after stimulation with EmagSA in immunized rabbits, indicated that protection against E. magna was established due to an effective local immune response. In particular, the exogenous proteins expressed by our transgenic $E$. magna induced cellular immunity in MLN as well. Our findings confirmed the capacity of transgenic rabbit coccidia to introduce foreign antigens into the rabbit immune system and showed the prospect of transgenic rabbit coccidia as a live vaccine vector.

\section{REFERENCES}

1. Duszynski DW, Couch L. The Biology and Identification of the Coccidia (Apicomplexa) of Rabbits of the World. London: Elsevier (2013).

2. Smith NC, Lunden A, Conraths FJ, Chapman HD. Control of coccidiosis into the next millennium. Parasitol Today (1998) 14:215-8. doi:10.1016/ S0169-4758(98)01238-1

3. Pakandl M. Coccidia of rabbit: a review. Folia Parasitol (Praha) (2009) 56:153-66. doi:10.14411/fp.2009.019

4. Blake DP, Tomley FM. Securing poultry production from the ever-present Eimeria challenge. Trends Parasitol (2014) 30:12-9. doi:10.1016/j.pt.2013. 10.003

5. Kelleher M, Tomley FM. Transient expression of beta-galactosidase in differentiating sporozoites of Eimeria tenella. Mol Biochem Parasitol (1998) 97:21-31. doi:10.1016/S0166-6851(98)00128-5

6. Yan W, Liu X, Shi T, Hao L, Tomley FM, Suo X. Stable transfection of Eimeria tenella: constitutive expression of the YFP-YFP molecule throughout the life cycle. Int J Parasitol (2009) 39:109-17. doi:10.1016/j/ijpara. 2008.06.013

7. Qin M, Liu XY, Tang XM, Suo JX, Tao GR, Suo X. Transfection of Eimeria mitis with yellow fluorescent protein as reporter and the endogenous development of the transgenic parasite. PLoS One (2014) 9:e114188. doi:10.1371/journal. pone. 0114188

8. Li Z, Tang X, Suo J, Qin M, Yin G, Liu X, et al. Transgenic Eimeria mitis expressing chicken interleukin 2 stimulated higher cellular immune response in chickens compared with the wild-type parasites. Front Microbiol (2015) 6:533. doi:10.3389/fmicb.2015.00533

9. Tang X, Yin G, Qin M, Tao G, Suo J, Liu X, et al. Transgenic Eimeria tenella as a vaccine vehicle: expressing TgSAG1 elicits protective immunity against Toxoplasma gondii infections in chickens and mice. Sci Rep (2016) 6:29379. doi:10.1038/srep29379

10. Tang X, Liu X, Tao G, Qin M, Yin G, Suo J, et al. “Self-cleaving” 2A peptide from porcine teschovirus-1 mediates cleavage of dual fluorescent proteins in transgenic Eimeria tenella. Vet Res (2016) 47:68. doi:10.1186/s13567016-0351-z

11. Jing F, Yin G, Liu X, Suo X, Qin Y. Large-scale survey of the prevalence of Eimeria infections in domestic rabbits in China. Parasitol Res (2012) 110:1495-500. doi:10.1007/s00436-011-2653-4

12. Licois D, Coudert P, Drouet-Viard F, Boivin M. Eimeria magna: pathogenicity, immunogenicity and selection of a precocious line. Vet Parasitol (1995) 60:27-35. doi:10.1016/0304-4017(94)00768-8

13. Pakandl M, Eid AN, Licois D, Coudert P. Eimeria magna Pérard, 1925: study of the endogenous development of parental and precocious strains. Vet Parasitol (1996) 65:213-22. doi:10.1016/S0304-4017(96)00975-2

14. Close B, Banister K, Baumans V, Bernoth EM, Bromage N, Bunyan J, et al. Recommendations for euthanasia of experimental animals: part 2. DGXT of the European Commission. Lab Anim (1997) 31(1):1-32.

15. Liu X, Shi T, Ren H, Su H, Yan W, Suo X. Restriction enzyme-mediated transfection improved transfection efficiency in vitro in Apicomplexan parasite Eimeria tenella. Mol Biochem Parasitol (2008) 161:72-5. doi:10.1016/ j.molbiopara.2008.06.006

\section{AUTHOR CONTRIBUTIONS}

XS, GT, and TS designed this study. GT and TS carried out the experiments with the help of XMT, YW, CL, JS, XLT, and XL. XS supervised the study implementation. GT drafted the manuscript. TS and DD contributed to the revision of the manuscript. All the authors read and approved the final version of the manuscript.

\section{FUNDING}

This research was supported by China Agricultural Research System (CARS-44) and the National Natural Science Foundation of China (31472180, 31472182, and 31330076).

16. Kim JH, Lee SR, Li LH, Park HJ, Park JH, Lee KY, et al. High cleavage efficiency of a $2 \mathrm{~A}$ peptide derived from porcine teschovirus-1 in human cell lines, zebrafish and mice. PLoS One (2011) 66(4):e18556. doi:10.1371/journal. pone.0018556

17. Yin G, Liu X, Zou J, Huang X, Suo X. Co-expression of reporter genes in the widespread pathogen Eimeria tenella using a double-cassette expression vector strategy. Int J Parasitol (2011) 41:813-6. doi:10.1016/j.ijpara. 2011.04.001

18. Long PL, Millard BJ, Joyner LP, Norton CC. A guide to laboratory techniques used in the study and diagnosis of avian coccidiosis. Folia Vet Lat (1976) 6:201.

19. Fetterer RH, Barfield RC. Characterization of a developmentally regulated oocyst protein from Eimeria tenella. J Parasitol (2003) 89:553-64. doi:10.1645/ GE-3159

20. Schmittgen T, Livak K. Analyzing real-time PCR data by the comparative $C_{T}$ method. Nat Protoc (2008) 6:1001-8. doi:10.1038/nprot.2008.73

21. Speer CA, Hammond DM, Youssef NN, Danforth HD. Fine-structural aspects of macrogametogenesis in Eimeria magna. J Protozool (1973) 20:274-81. doi:10.1111/j.1550-7408.1973.tb00875.x

22. Wallach M. Role of antibody in immunity and control of chicken coccidiosis. Trends Parasitol (2010) 26:382-7. doi:10.1016/j.pt.2010.04.004

23. Wang JL, Huang SY, Behnke MS, Chen K, Shen B, Zhu XQ. The past, present, and future of genetic manipulation in Toxoplasma gondii. Trends Parasitol (2016) 32:542-53. doi:10.1016/j.pt.2016.04.013

24. Tellier G, Lenne A, Cailliau-Maggio K, Cabezas-Cruz A, Valdes JJ, Martoriati A, et al. Identification of Plasmodium falciparum translation initiation eIF2beta subunit: direct interaction with protein phosphatase type 1. Front Microbiol (2016) 7:777. doi:10.3389/fmicb.2016.00777

25. Vinayak S, Pawlowic MC, Sateriale A, Brooks CF, Studstill CJ, Bar-Peled Y, et al. Genetic modification of the diarrhoeal pathogen Cryptosporidium parvum. Nature (2015) 523:477-80. doi:10.1038/nature14651

26. Shi T, Tao G, Bao G, Suo J, Hao L, Fu Y, et al. Stable transfection of Eimeria intestinalis and investigation of its life cycle, reproduction and immunogenicity. Front Microbiol (2016) 7:807. doi:10.3389/fmicb.2016.00807

27. Wurm FM. Production of recombinant protein therapeutics in cultivated mammalian cells. Nat Biotechnol (2004) 22:1393-8. doi:10.1038/nbt1026

28. Renaux S, Quéré P, Buzoni-Gatel D, Sewald B, Le Vern Y, Coudert P, et al. Dynamics and responsiveness of T-lymphocytes in secondary lymphoid organs of rabbits developing immunity to Eimeria intestinalis. Vet Parasitol (2003) 110:181-95. doi:10.1016/S0304-4017(02)00305-9

Conflict of Interest Statement: The authors declare that the research was conducted in the absence of any commercial or financial relationships that could be construed as a potential conflict of interest.

Copyright (c) 2017 Tao, Shi, Tang, Duszynski, Wang, Li, Suo, Tian, Liu and Suo. This is an open-access article distributed under the terms of the Creative Commons Attribution License (CC BY). The use, distribution or reproduction in other forums is permitted, provided the original author(s) or licensor are credited and that the original publication in this journal is cited, in accordance with accepted academic practice. No use, distribution or reproduction is permitted which does not comply with these terms. 